\title{
Challenges of Establishing a de novo Consultation-Liaison Psychiatry Service in a Tertiary Hospital \\ Practical considerations and challenges
}

Nawal N. Al Mahyijari' and "Ibrahim M. Inuwa ${ }^{2}$

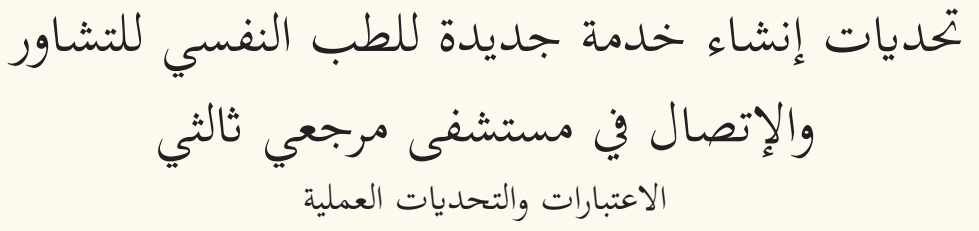

نوال الدحيجري و إبراهيم محمد إنوة

ABSTRACT: Consultation-liaison psychiatry (CLP), also known as psychosomatic medicine, is a subspecialty of psychiatry that focuses on the care of patients with mental health disorders and general medical/surgical conditions. Integrating CLP services facilitates diagnosis and management of patients with complex comorbidities. This article aimed to report the practical considerations and challenges associated with establishing a de novo CLP service in a tertiary hospital in the Gulf region. This includes discussing the rationale and clinical and educational benefits as well as the resources required for establishing a CLP service.

Keywords: Psychiatry; Medical Education.

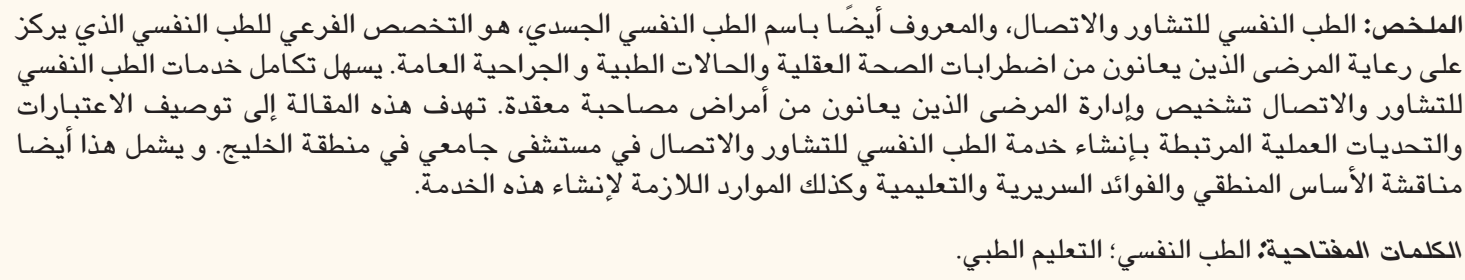

C NSULTATION-LIAISON PSYCHIATRY (CLP) has been described as "the guardian of holistic approach to the patient". This emphasises its vital role in the care of patients admitted to any general hospital. Other researchers have stated that there is "no health without mental health" and that integrating therapy into the bio-psychosocial model should be the main objective of CLP services rather than administration of psychiatric treatment only. ${ }^{2,3}$

In many situations, health professionals have patients with existing physical comorbidities and/ or mental health disorders. ${ }^{4}$ In hospitals where there are no CLP services, such situations place a heavy economic burden on finite hospital resources and prolong the suffering of affected patients. ${ }^{2}$ Studies have observed that the existence of mental health disorders, even when sub-clinical in patients with a medical illness, could lead to longer hospital stays and worse health outcomes. ${ }^{5-7}$
Establishing de novo CLP services has been studied in various parts of the world. ${ }^{3,8-13}$ For example, De Giorgio et al's study provides evidence that supports establishing inpatient CLP services. ${ }^{3}$ They found that implementing inpatient CLP in medical/surgical/ obstetrics-gynaecology wards with an emphasis on clinimetric approaches (i.e. "the development of instruments to measure multiple constructs with a single index") rather than psychometric approaches (i.e. the development of "instruments that measure a single construct using multiple items"), especially in a multidisciplinary context and specifically for complex cases, yields holistic health benefits. ${ }^{3,14}$ The availability of CLP services shortens length of hospital stay and facilitates a more coordinated approach when dealing with complex cases. ${ }^{3}$ As a result, several countries, including some in the Gulf region, have started to implement inpatient CLP services in general hospitals. ${ }^{11,12}$ 
Inpatient CLP services are well developed in many countries around the world including Europe and North America where continuous scientific exchanges have a positive impact on education, healthcare practice and, ultimately, patients outcomes. ${ }^{15-17}$ In Oman specifically, and in the Gulf region generally, there are no comprehensive statistics available on overall levels of activity, or resources allocated to CLP services in general hospitals. General psychiatrists who can facilitate this CLP services are needed in the Ministry of Health general hospitals. There is an urgent need to focus resources within and between different Gulf countries with the aim of developing CLP scientific exchange. Therefore, the main objective of this article is to discuss the practical considerations and challenges associated with establishing a de novo CLP service in a tertiary hospital, including the rationale and clinical and educational benefits as well as the resource requirements for establishing such a service.

\section{The Rationale}

The prevalence of comorbid mental health disorders among patients in general hospitals is extremely high; a questionnaire-based study revealed nearly one in five patients admitted for acute medical service required psychiatric consultation. ${ }^{9}$ Furthermore, many of these cases are typically undiagnosed and, therefore, not treated. ${ }^{9}$ The absence of effective intervention may affect patients' response to medical treatment, quality of life, utilisation of services, length of hospital stays and increase the cost of health care. ${ }^{8,13}$ Improvement in diagnosis and management of mental health conditions in general hospitals can significantly reduce the scale and cost of these problems. ${ }^{9,14}$ Given these epidemiological considerations, there is a need for dedicated and specialised inpatient CLP services in general hospitals. ${ }^{9}$

Currently, there is a trend towards emphasising the importance of collaboration among health professionals in the treatment of patients with complex comorbidities. ${ }^{9} 12$ This includes combined medicalmental health consultation and longitudinal care in order to provide integrated and holistic care. Therein lies a vital role for CLP to help bridge the gap between the physical and mental health needs of patients and reducing mental health stigma. ${ }^{13}$ Therefore, inpatient CLP service has numerous advantages in assessing mental health and physical comorbidities accurately and educating clinicians about the impact of symptoms on psychological/physical health and how to manage them. ${ }^{11,13}$

\section{Clinical Benefits}

An important function of CLP service is the identification of patients that require complex biopsychosocial intervention such as patients with delirium, deliberate self-harm, substance abuse and unexplained physical complaints. These patients require an effective interdisciplinary team approach for their care. Such patients should be identified upon admission or early during their hospitalisation in order to optimise the coordination and management necessary for their care. Importantly, validated tools, such as Complexity prediction instrument (COMPRI) and INTERMED, that are useful in identifying the needs of these complex cases either at the time of admission or among the outpatient population should be made available to CLP teams. ${ }^{15,18}$

\section{Educational Benefits}

With respect to medical education, there is authoritative evidence-based information that supports integrating inpatient CLP into medical and surgical department. ${ }^{8}$ Liaison-psychiatry has been a core-training requirement of residency programmes in certain parts of the world. 8 ,10 Medical students who were exposed to CLP training had greater knowledge of and a positive attitude towards psychiatry, which might encourage recruitment to the profession. ${ }^{8,10}$ Inpatient CLP professionals may play a major role in collaborating with the medical team to provide a strong focus on education and training of various medical/surgical teams. This training could include how to implement basic psychotherapeutic interventions that could facilitate patients' coping with the stress of medical illness. On a larger community-wide scale, CLP services could also perform an important bridge function between general practice and mental healthcare thereby reducing stigma around mental health and improving the training of general practitioners. ${ }^{19}$

In addition, there are many areas in medicine that lend themselves to developing exciting research questions within the context of CLP. Educational benefits could include future research on topics such as the phenomenology of somatisation disorders (somatoform disorders in the International Classification of Diseases-10), interactions of biopsychosocial factors and the effectiveness of CLP interventions.

\section{Resource Requirements for Establishing a CLP Service}

A full-time CLP specialist is essential in establishing clear guidelines and in determining what constitutes optimal interventions for a patient in need of complex 
care. In addition, psychologists and social workers will greatly contribute to patients' quality of life and decrease the burden of care of this highly complex patient population. Furthermore, a liaison nurse is an essential professional necessary for a well-functioning interdisciplinary CLP service., ${ }^{911}$ A liaison nurse provides support to nursing staff in medical/surgical departments inside the hospital and facilitates the organisation of mental health follow-ups on discharge or transfers to psychiatric facilities. Finally, it would be necessary to ensure adequate physical resources to support the CLP team. A suggested flowchart of how such service could be embedded in the care of patients is illustrated in Figure 1.

\section{Challenges in Establishing CLP Service}

Baseline staffing of CLP services in general hospitals have been previously described. ${ }^{20}$ Multiple factors that determine efficient sizing of a CLP service include the number of beds, nature of illnesses, availability of on-site trainees and financial constraints. While it is easy to find consensus that CLP implementation is important to ensure the adequate diagnosis and treatment of medically ill patients with mental health disorders, the provision of such CLP services face a number of challenges.

Evidence suggests that in hospitals where CLP service exists, referral rates are very low..$^{21,22}$ This may

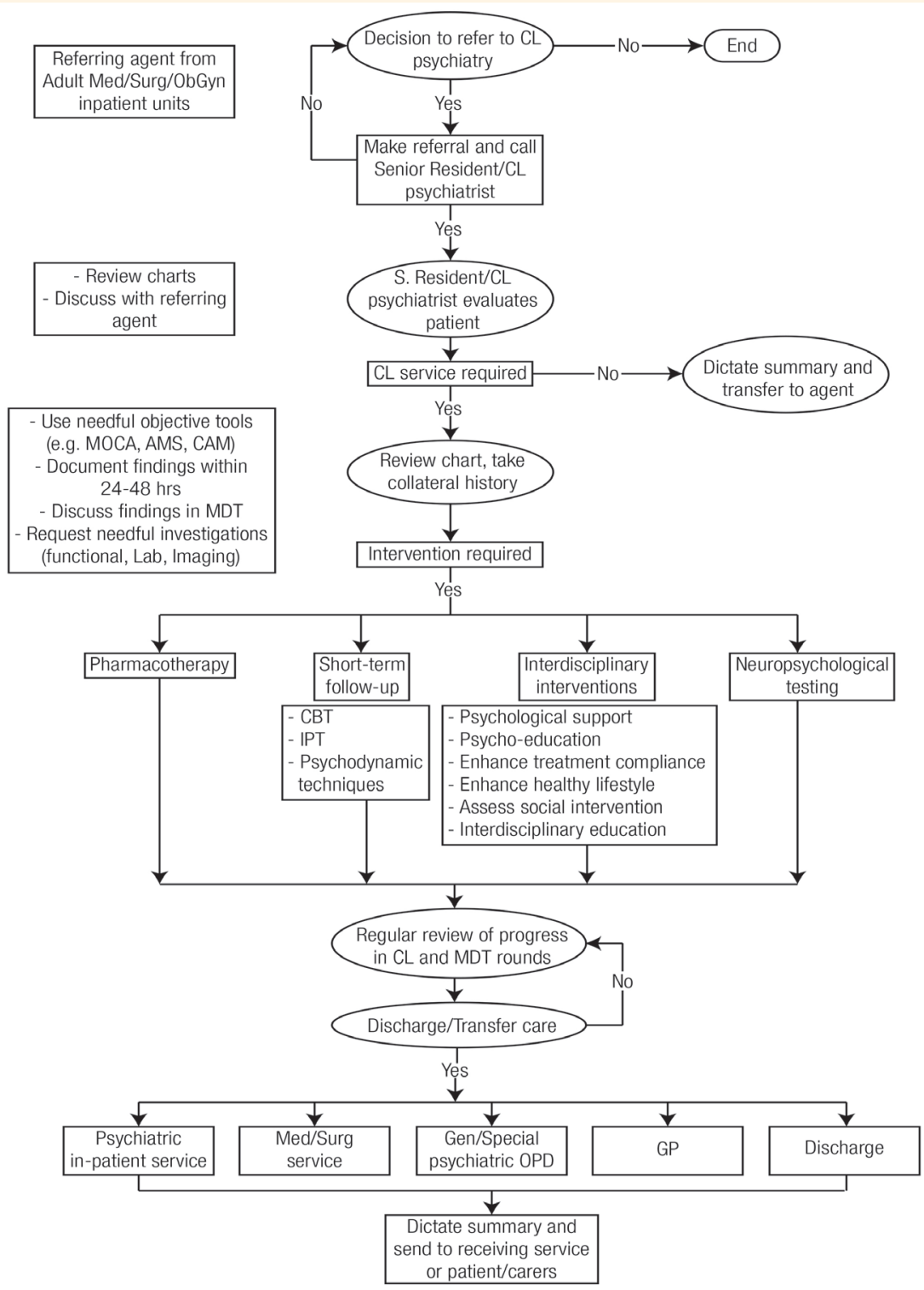

Figure 1: Suggested flowchart of how a consultation-liaison psychiatry service could be embedded in the care of hospital patients. Med = medical; Surg = surgical; ObGyn = obstetrics and gynaecology; $C L=$ consultation liaison; $S .=$ senior; $M O C A=$ Montreal Cognitive Assessment; AMS = Arabic Memory Screening test; $C A M=$ Confusion Assessment Method; $M D T=$ multidisciplinary team; Lab = laboratory; $C B T=$ cognitive behavioural therapy; $I P T=$ interpersonal psychotherapy; Gen = general; $O P D=$ outpatient department; $G P=$ general practitioner. 
be due to the doctors on medical/surgical wards not noticing a disturbance in a patient's emotional state as well as many physicians being reluctant to use psychiatric services. ${ }^{21,22}$ Where such CLP services exist, they are often encumbered by unpredictable schedules and variable numbers of consultations. In addition, developing countries, including the Gulf countries, are facing many other challenges including insufficient training of medical and paramedical staff, funding for clinical practice and research, coordination with complementary and alternative medicine practitioners and effective networking with policy makers in combating the epidemic of non-communicable diseases including mental health disorders. ${ }^{19}$

To overcome these challenges, evidence of the potential benefits of CLP services on issues such as length of stay and quality of patient care should be well articulated. This may convince hospital administrators and decision-makers to support the creation of a CLP service. In addition, mental health practitioners should share data on mental health needs to gain a more comprehensive national and international perspective that can be used to convince all stakeholders in regards to the acceptable benchmarks for CLP services. ${ }^{16}$

\section{Conclusion}

While there are various challenges in establishing a CLP service, there are also significant benefits in integrating inpatient CLP services in the Gulf region. It facilitates timely diagnosis and management of complex cases. The liaison service helps to establish professional contact with medical/surgical and nursing colleagues, reduce mental health stigma, broaden the understanding of psychological reactions to physical illness and of psychogenic disorders, reduce unnecessary investigations and provide more comprehensive patient care. In addition, CLP services could facilitate early detection of mental health problems in patients and provide support to the medical/surgical staff in caring for such patients.

\section{References}

1. Ajiboye PO. Consultation-liaison psychiatry: The past and the present. Afr J Med Med Sci 2007; 36:201-5.

2. Smith GC. From consultation-liaison psychiatry to integrated care for multiple and complex needs. Aust N Z I Psychiatry 2009; 43:1-12. https://doi.org/10.1080/00048670802534358.

3. De Giorgio G, Quartesan R, Sciarma T, Giulietti M, Piazzoli A, Scarponi L, et al. Consultation-liaison psychiatry-from theory to clinical practice: An observational study in a general hospital. BMC Res Notes 2015; 8:475. https://doi.org/10.1186/s13104015-1375-6.

4. Smith GC. Challenges for psychotherapy and psychoanalysis. Aust J Psychother 2006; 25:32-55.
5. Ormel J, Vonkorff F, Oldenhinkel AJ, Simon G, Tiemens BG, Ustün TB. Onset of disability in depressed and non-depressed primary care patients. Psychol Med 1999; 29:847-53. https://doi.org/10.1017/s0033291799008600.

6. Saravay SM, Lavin M. Psychiatric comorbidity and length of stay in the general hospital. A critical review of outcome studies. Psychosomatics 1994; 35:233-52. https://doi.org/10.1016/s00333182(94)71772-2

7. Chilsholm D, Diehr P, Knapp M, Patrick D, Treglia M, Simon G, et al. Depression status, medical comorbidity and resource costs. Evidence from an international study of major depression in primary care (LIDO). Br J Psychiatry 2003; 183:121-31 https://doi.org/10.1192/bjp.183.2.121.

8. Desan PH, Zimbrean PC, Weinstein AJ, Bozzo JE, Sledge WH. Proactive psychiatry consultation services reduce length of stay for admissions to an inpatient medical team. Psychosomatics 2011; 52:513-20. https://doi.org/10.1016/j.psym.2011.06.002.

9. Lloyd GG. Liaison psychiatry from a British perspective. Gen Hosp Psychiatry 1980; 2:46-51. https://doi.org/10.1016/01638343(80)90106-1.

10. Kurosawa H, Iwasaki Y, Watanabe N, Nakamura K, Kishi Y, Huse R. The practice of consultation-liaison psychiatry in Japan. Gen Hosp Psychiatry 1993; 15:160-5. https://doi.org/10.1016/0 163-8343(93)90119-9.

11. Fido AA, Al Mughaiseeb A. Consultation liaison psychiatry in a Kuwaiti general hospital. Int J Soc Psychiatry 1988; 35:274-9. https://doi.org/10.1177/002076408903500308.

12. Aljarad AM, Al Osaimi FD, Al Huthail YR. Accuracy of psychiatric diagnoses in consultation liaison psychiatry. J T U Med Sci 2008; 3:123-8. https://doi.org/10.1016/S1658-3612(08)70061-1.

13. Andreoli PB, Citero Vde A, Mari Jde J. A systematic review of studies of the cost-effectiveness of mental health consultationliaison interventions in general hospitals. Psychosomatics 2003; 44:499-507. https://doi.org/10.1176/appi.psy.44.6.499.

14. de Vet HCW, Terwee CB, Bouter LM. Clinimetrics and psychometrics: Two sides of the same coin. J Clin Epidemiol 2003; 56:1146-7. https://doi.org/10.1016/j.jclinepi.2003.08.010.

15. Huyse FJ, de Jonge P, Slaets JP, Herzog T, Lobo A, Lyons JS, et al. COMPRI--An instrument to detect patients with complex care needs: Results from a European study. Psychosomatics 2001; 42:222-8. https://doi.org/10.1176/appi.psy.42.3.222.

16. Kunkel EJ, Del Busto E, Kathol R, Stern TA, Wise TN, Stoddard Fr 2nd, et al. Physician staffing for the practice of psychosomatic medicine in general hospitals: A pilot study. Psychosomatics 2010; 51:520-7. https://doi.org/10.1016/s0033-3182(10)70746-5.

17. Deter HC, Orth-Gomér K, Wasilewski B, Verissimo R. The European Network on Psychosomatic Medicine (ENPM) History and future directions. Biopsychosoc Med 2017; 11:3. https://doi.org/10.1186/s13030-016-0086-0.

18. Huyse FJ, Lyons JS, Stiefel FC, Slaets JP, de Jonge P, Fink P, et al. "INTERMED": A method to assess health service needs. I. Development and reliability. Gen Hosp Psychiatry 1999; 21:39-48. https://doi.org/10.1016/s0163-8343(98)00057-7.

19. Chandrashekar CR, Math SB. Psychosomatic disorders in developing countries: Current issues and future challenges. Curr Opin Psychiatry 2006; 19:201-6. https://doi.org/10.1097/01.yco.0 000214349.46411.6a.

20. Holmes A, Handrinos D, Theologus E, Salzberg M. Service use in consultation-liaison psychiatry: Guidelines for baseline staffing. Australas Psychiatry 2011; 19:254-8. https://doi.org/1 $0.3109 / 10398562.2011 .562505$.

21. Alqataybi $\mathrm{H}$, Almaqbali $\mathrm{M}$, Asiri WA, Martin R, Alsinawi $\mathrm{H}$. Patterns of referrals to consultation-liaison psychiatry in a tertiary care hospital in Oman. Arab J Psychiatry 2018; 29:17-23. https://doi.org/10.12816/0046438.

22. Chen KY, Evans R, Larkins S. Why are hospital doctors not referring to consultation-liaison psychiatry? - A systemic review. BMC Psychiatry 2016; 16:390. https://doi.org/10.1186/ s12888-016-1100-6. 